\title{
A unique epigenomic landscape defines the characteristics and differentiation potentials of glioma stem cells
}

\author{
Bing Yao ${ }^{*}$ and Peng Jin ${ }^{*}$
}

\begin{abstract}
A new study reveals comprehensive and unique epigenetic properties of glioma stem cells, leading to novel molecular insights and therapeutic potentials toward glioblastoma multiforme treatment.
\end{abstract}

\section{Introduction}

Glioblastoma multiforme (GBM), also known as glioblastoma, is one of the most aggressive and infiltrative tumors in the central nervous system (CNS). The 5-year survival rate of patients with GBM is less than $5 \%$, and they have a poor prognosis for recovery [1]. Therefore, new, effective therapeutic approaches to treat GBM are urgently needed. Despite substantial progress over the past decades, our understanding of GBM pathology at the molecular level is still largely lacking, owing to its heterogeneous nature with unique genetic and epigenetic alterations [2].

Neurogenesis generates various functional neural cell types from multipotent neural stem cells (NSCs) in the mammalian CNS, which participate in specific neural activities and responses [3]. The concept of the cancer stem cell is based on the fact that a subpopulation of glioma cells share common features with NSCs, including self-renewal and the differentiation potential to glial and neuronal cells [4]. This model explains how cancer stem cells could serve as driving forces of cancer malignancy and recurrence. In vitro, cultured glioma stem cells (GSCs) that were derived from primary GBM cells possess many features of NSCs and remarkably have characteristics resembling those of GBM cells, such as genetic aberrations, global transcriptome profiles, and tumorigenic potentials [5]. Therefore, GSCs are an

\footnotetext{
* Correspondence: bing.yao@emory.edu; peng.jin@emory.edu Department of Human Genetics, Emory University School of Medicine, Atlanta, GA 30322, USA
}

(c) The Author(s). 2018 Open Access This article is distributed under the terms of the Creative Commons Attribution 4.0 International License (http://creativecommons.org/licenses/by/4.0/), which permits unrestricted use, distribution, and reproduction in any medium, provided you give appropriate credit to the original author(s) and the source, provide a link to the Creative Commons license, and indicate if changes were made. The Creative Commons Public Domain Dedication waiver (http://creativecommons.org/publicdomain/zero/1.0/) applies to the data made available in this article, unless otherwise stated. excellent therapeutic target for GBM treatment. The lack of complete and high-quality epigenetic and transcriptome maps in GSCs hinders our understanding of the underlying critical differences at the transcriptional level between malignant GSCs and normal NSCs, as well as the epigenetic machinery involved in regulating their proliferation and differentiation.

In this issue of Genome Biology, Zhou et al. take on this vital challenge to generate comprehensive genomewide epigenomic profiling for various forms of cytosine modifications of DNA at single-base resolution and signature histone modifications of enhancers and transcriptomes of GSCs isolated from patient-derived xenografts, with NSCs isolated from fetal brains as controls [6].

\section{Epigenetic dysregulations in GSCs}

Covalent modifications of DNA at the 5-carbon position of cytosine, such as 5 -methylcytosine $(5 \mathrm{mC})$, play significant epigenetic roles in the mammalian brain. $5 \mathrm{mC}$ is thought to be a static and irreversible modification. However, the recent discovery that Ten-eleven translocation (TET) proteins, viewed as $5 \mathrm{mC}$ "erasers," catalyze the conversion of $5 \mathrm{mC}$ to 5-hydroxymethylcytosine $(5 \mathrm{hmC})$, providing a new perspective on the plasticity of cytosine modification. Subsequent studies revealed that TET proteins further oxidize $5 \mathrm{hmC}$ to 5 -formylcytosine (5fC) and 5-carboxylcytosine $(5 \mathrm{caC})$, which are converted to cytosine through DNA repair pathways by thymine-DNA glycosylase (TDG). Cytosine modifications are dynamically and precisely regulated during neurodevelopment and neuronal functions, to spatially and temporally control key gene expression. Thus their dysregulations are linked to many brain disorders, including GBM $[7,8]$.

In this study, the authors first focused on connecting the expression of DNA modification machinery and the global levels of these modifications in GSCs. There was 
a notable depletion of certain DNA modifications, such as $5 \mathrm{hmC}$ and $5 \mathrm{fC}$, compared with NSCs, indicating a global DNA modification perturbation in GSCs. Interestingly, they found a significant negative correlation of $5 \mathrm{mC}$ modification with TET2 and TDG expression, and a positive correlation between TET2 expression and $5 \mathrm{fC}$ modification, in GSCs. On the basis of the findings by Zhou et al., it is plausible that TET2 sits at the center stage of maintaining DNA modification homeostasis in normal brain function, and its dysregulation could result in the profound alteration of neuroepigenetic landscaping, contributing to GBM tumorigenesis.

\section{GSCs display global transcriptome alterations}

Transcriptome profiling of GSCs by Zhou et al. identified the upregulation of HOX genes, which potentially account for the GSC proliferation, and the downregulation of genes involved in apoptosis, growth inhibition, and neural development. Also, several highly conserved downregulated miRNAs were observed and potentially linked to patient survival. These findings at the molecular level identify the characteristics of GSCs and provide solid foundations for future mechanistic exploration.

The authors also performed systematic, integrated analyses of genome-wide DNA modification dynamics, enhancer activities, and gene expression changes to define the epigenetic mechanisms that account for the transcriptome alteration in GSCs. While GSCs share a significant portion of enhancers with NSCs, as defined by their histone marks, many GSC-specific active enhancers were identified that potentially link to gene misregulation. These enhancer-shifting patterns indicate that GSCs could lose a set of NSC-specific enhancer footprints and gain a new set of cis-regulatory regions to influence tumorigenic transcription ectopically. In addition, global loss of $5 \mathrm{mC}$ and $5 \mathrm{hmC}$ accompanied by gain of $5 \mathrm{fC}$ or $5 \mathrm{caC}$ were found in GSCs, which could contribute to gene misregulation by recruiting a distinct set of "readers." A recent observation suggests that TET2 plays a critical role in further converting $5 \mathrm{hmC}$ to $5 \mathrm{fC} / 5 \mathrm{caC}$, as depletion of TET2 results in the accumulation of $5 \mathrm{hmC}$ in promoters and gene bodies [7]. The upregulation of TET2 and accumulation of $5 \mathrm{fC} / 5 \mathrm{caC}$ seen in this study support this notion. Further research on the molecular mechanisms dictating TET2 differential activities toward $5 \mathrm{mC} / 5 \mathrm{hmC}$ or $5 \mathrm{hmC} / 5 \mathrm{fC}$ conversion is necessary to confirm this. Nonetheless, these integrated analyses by Zhou et al. highlight the epigenetic causality of GSC-associated transcriptional alteration and demonstrate the significant roles of TET proteins during this process.

\section{Unique epigenetic profiles associated with GSC differentiation}

Asymmetrical differentiation of GSCs could contribute to the tumor mass, and terminal differentiation of these GSCs could eliminate these cell populations for ideal therapeutic outcomes. Epigenetic changes of GSC and NSC differentiation were profiled in parallel, to understand the precise epigenetic alterations during GSC differentiation relative to that of NSCs. While NSC differentiation acquires developmentally programmed DNA modifications during differentiation, the DNA modifications in the GSC genome appear to be more loosely or randomly controlled, which may explain the fact that GSCs possess greater plasticity regarding various endogenous and extracellular differentiation cues for tumorigenesis. Consistent with this, the GSCs with differential TET2 or TET3 expression levels clearly displayed distinctive responses to chemotherapeutic agents, providing important mechanistic insights of current therapeutic challenges. Given the central role of TET proteins in tumorigenesis, it is possible that they may serve as therapeutic targets for GBM patients. Owing to technical challenges and poor antibody quality, the genome-wide distributions of TET proteins in GSCs are still unknown, and it would be important to correlate the binding dynamics of epigenetic machinery with their marks to gain an improved understanding of their molecular mechanisms in order to further therapeutic efforts.

\section{Conclusions}

The present study presents the first detailed characterization of the GSC epigenome, including DNA methylation/demethylation and histone modifications associated with distal regulatory elements. However, the GSCs used in this study were isolated from GBM patient-derived xenografts. The development of single-cell-transcriptome and epigenome analyses will enable the further delineation of epigenomic changes associated with primary GBM tumors and GSCs, as well as tumor organoids. These results will provide further insight into epigenomic alterations associated with GBM and GSCs. More importantly, it will be critical to determine the functionality of causal loci with epigenetic alterations associated with GBM, which may lead to identification of novel therapeutic targets and pathway for GBM.

\section{Funding}

Research in PJ's laboratory is supported in part by NIH grants (NS051630, NS079625, MH102690, NS097206 and AG052476).

Authors' contributions

BY and PJ wrote the article and read and approved the final manuscript. 


\section{Competing interests}

The authors declare that they have no competing interests.

\section{Publisher's Note}

Springer Nature remains neutral with regard to jurisdictional claims in published maps and institutional affiliations.

\section{Published online: 10 April 2018}

\section{References}

1. Ostrom QT, Bauchet L, Davis FG, Deltour I, Fisher JL, Langer CE, et al. The epidemiology of glioma in adults: a "state of the science" review. NeuroOncology. 2014;16:896-913.

2. Liu A, Hou C, Chen H, Zong X, Zong P. Genetics and epigenetics of glioblastoma: applications and overall incidence of $\mathrm{IDH} 1$ mutation. Front Oncol. 2016;6:16

3. Ming $\mathrm{GL}$, Song $\mathrm{H}$. Adult neurogenesis in the mammalian brain: significant answers and significant questions. Neuron. 2011;70:687-702.

4. Fine HA. Glioma stem cells: not all created equal. Cancer Cell. 2009;15:247-9.

5. Lee J, Kotliarova S, Kotliarov Y, Li A, Su Q, Donin NM, et al. Tumor stem cells derived from glioblastomas cultured in bFGF and EGF more closely mirror the phenotype and genotype of primary tumors than do serum-cultured cell lines. Cancer Cell. 2006;9:391-403.

6. Zhou D, Alver BM, Li S, Hlady RA, Thompson JJ, Schroeder MA, et al.

Distinctive epigenomes characterize glioma stem cells and their response to differentiation cues. Genome Biol. 2018:19:43. https://doi.org/10.1186/ s13059-018-1420-6

7. Johnson KC, Houseman EA, King JE, von Herrmann KM, Fadul CE, Christensen BC. 5-hydroxymethylcytosine localizes to enhancer elements and is associated with survival in glioblastoma patients. Nat Commun. 2016; 7:13177.

8. Li X, Yao B, Chen L, Kang Y, Li Y, Cheng Y, et al. Ten-eleven translocation 2 interacts with forkhead box $\mathrm{O} 3$ and regulates adult neurogenesis. Nat Commun. 2017:8:15903. 